\title{
Cytokeratin 5/14-positive breast cancer: true basal phenotype confined to BRCA1 tumors
}

\author{
Mervi Laakso ${ }^{1,2}$, Niklas Loman ${ }^{3}$, Åke Borg ${ }^{3}$ and Jorma Isola ${ }^{2}$ \\ ${ }^{1}$ Department of Pathology, Seinäjoki Central Hospital, Seinäjoki, Finland; ${ }^{2}$ Institute of Medical Technology, \\ University of Tampere, Tampere, Finland and ${ }^{3}$ Department of Oncology, University of Lund, Lund, Sweden
}

\begin{abstract}
Breast ducts contain two types of epithelial cells, inner luminal cells and outer basal/myoepithelial cells. These cells can be distinguished by their immunophenotype. Cytokeratins (CKs) 8 and 18 are expressed in the luminal layer, whereas CK5/14 and the transcription factor p63 characterize the basal epithelial layer. We studied a population-based cohort of 288 sporadic ductal invasive cancers and found $9 \%$ positive for CK5/14 and $4 \%$ positive for p63. Using a highly sensitive polymer-based immunohistochemical staining, all sporadic tumors were positive for the luminal $C K 8 / 18$, including those positive for CK5/14. Pairs of primary tumors and metastases $(n=38)$ were always concordant for CK5/14 expression. The majority of the CK5/14-positive cases were of histologic grade III $(P=0.0007)$ and steroid hormone receptor negative $(P<0.0001)$. CK5/14 expression was inversely associated with HER-2 oncogene amplification, but only in the subgroup of estrogen receptornegative tumors $(P=0.007)$. In a separate set of 42 hereditary breast cancers, the majority $(78 \%)$ of the $B R C A 1$ associated tumors, but only one of 15 BRCA2-associated tumors was positive for CK5/14. In contrast to sporadic CK5/14-positive tumors, BRCA1-associated tumors displayed less intense CK8/18 staining, including some truly CK5/14-positive CK8/18-negative cases. These results suggest that CK5/14-positive sporadic breast cancers arise from glandularly committed progenitor cells rather than true CK8/18-negative basal cells. Modern Pathology (2005) 18, 1321-1328. doi:10.1038/modpathol.3800456; published online 1 July 2005
\end{abstract}

Keywords: basal phenotype; BRCA1; BRCA2; cytokeratin; HER-2; immunohistochemistry; oncogene; progenitor cell

The degree of differentiation and functional characteristics of epithelial cells giving rise to breast carcinoma have remained unclear. Most investigators have addressed breast carcinoma precursors by analyzing expression of cytokeratins (CKs) as differentiation markers, since their expression is thought to remain stable throughout carcinogenesis. ${ }^{1}$ In general, breast cancers are thought to arise from luminally differentiated epithelial cells, as evidenced by strong expression of CK8, CK18 and CK19, similar to the situation in the cells lining the lumen of normal breast ducts. ${ }^{1-3}$ A small fraction of breast cancers express CK5 together with its major partners CK14 and CK17,,4-7 which are normally found in the basal cell layer of the mammary duct. $^{1-3,8}$ For this reason, tumors expressing these CKs have been named 'basal-type' breast cancer. Breast cancers are generally thought to express

Correspondence: M Laakso, MSc, Department of Pathology, Seinäjoki Central Hospital, Hanneksenrinne 7, Seinäjoki 60220, Finland.

E-mail: mervi.laakso@epshp.fi

Received 17 February 2005; revised 24 May 2005; accepted 25 May 2005; published online 1 July 2005 either luminal $(\mathrm{CK} 8 / 18 / 19+)$ or basal $(\mathrm{CK} 5 / 14+)$ cytokeratins. ${ }^{5,9,10}$ However, some CK5/14- and CK8/ 18-coexpressing tumors have also been found..$^{2,4,6,8} \mathrm{It}$ has been proposed that tumors positive for CK5 originate from multipotent CK5-expressing progenitor cells,,$^{2,8,11,12}$ located between the basal/suprabasal and luminal cell layers in normal ducts. ${ }^{8}$ CK5positive progenitor epithelial cells can gradually differentiate towards glandular and myoepithelial lineages. ${ }^{2,8,11,12}$

Basal phenotype tumors represent a histologically poorly differentiated estrogen receptor (ER)-negative tumor subtype. ${ }^{4,6,7,9,10,13-16}$ The precise prevalence and clinicopathological characteristics of basal and luminal CK-expressing and -coexpressing tumors remain unclear. In particular, it is currently not known whether amplification of the HER-2 oncogene is characteristic of basal or luminal phenotype tumors, or whether there is no association. The results of some studies have suggested that basal phenotype tumors may express less HER-2 protein. ${ }^{7,16}$ These findings are supported by the results of gene expression microarray studies, which exclusively classify HER-2-expressing and basal phenotype tumors as separate entities. ${ }^{10,17,18}$ In contrast, 
Birmbaum et al ${ }^{9}$ have reported that HER-2 oncogene amplification would be associated with basal phenotype breast cancer. More coherent data is available on hereditary breast cancers in BRCA1 mutation carriers. These tumors have a high frequency of basal cell CK expression, ${ }^{18-20}$ but for BRCA2-associated tumors, no immunohistochemical CK expression data have been reported.

In addition to CKs, the nuclear transcription factor p63 is a newly discovered marker of basal and myoepithelial cells in normal breast. ${ }^{21-24}$ Its expression has been found in $10-12 \%$ of breast tumors, ${ }^{24-26}$ and it is associated with high grade, large tumor size, nodal metastasis and ER negativity. However, some investigators have found no p63 in invasive breast carcinomas. ${ }^{21,23}$ Thus, current data on p63 expression and its associations is controversial.

In the present work, we studied basal (CK5/14) and luminal (CK8/18/19) CK and p63 expression in a large population-based cohort of sporadic invasive ductal breast cancers as well as in tumors from a separate cohort of BRCA1 and BRCA2 germline mutation carriers. Our aim was to define the precise prevalence of basal phenotype breast tumors, and to examine clinicopathological correlations, with special emphasis on association with the HER-2 oncogene. For this purpose, we analyzed an additional set of 141 HER-2-amplified breast tumors.

\section{Materials and methods}

\section{Tumor Samples}

The study material consisted of a population-based cohort of 288 consecutive sporadic invasive ductal breast cancers derived from the archives of the Department of Pathology at Seinäjoki Central Hospital. Histopathological information was collected for each patient sample (including grade, tumor size, lymph node metastasis, ER, progesterone receptor (PR) and HER-2). Separate sets of 27 tumors from $B R C A 1$ germline mutation carriers, 15 tumors from BRCA2 germline mutation carriers (both from Department of Oncology, University of Lund, Lund, Sweden), 141 HER-2-amplified cancers and 38 pairs of primary and metastatic carcinomas (both from Department of Pathology, Seinäjoki Central Hospital, Seinäjoki, Finland and Institute of Medical Technology, University of Tampere, Tampere, Finland) were also studied. Mutation analyses of BRCA1 and BRCA2 have been described previously. ${ }^{27}$

To optimize and validate the basal phenotype immunostaining method, a subset $(n=101)$ of the tumors was prepared as five tissue microarray blocks, each containing cores $(1 \mathrm{~mm}$ diameter $)$ of carcinoma-containing tumor tissue. All samples were routinely formalin fixed and paraffin embedded. Sections were cut $(3 \mu \mathrm{m})$ and used for immunohistochemistry.

\section{Immunohistochemistry}

The slides were deparaffinized and rehydrated before pretreatment. Antibodies tested were against: CK5, clone XM26 (Novocastra, Newcastle upon Tyne, UK); CK14, clone LL002 (Novocastra); CK5/ 6, clone D5/16B4 (DakoCytomation, Glostrup, Denmark) and p63, clone 4A4+Y4A3 (Neomarkers, Fremont, CA, USA). Five different antigen retrieval pretreatments were compared for all antibodies using adjacent tissue microarray sections: protease (from Bacillus Licheniformis, Sigma-Aldrich, St Louis, MO, USA) treatment at $37^{\circ} \mathrm{C}$ for $3 \mathrm{~min} 30 \mathrm{~s}$, heat treatment in an autoclave at $103^{\circ} \mathrm{C}$ for $5 \mathrm{~min}$ with $0.05 \mathrm{M}$ Tris-HCl buffer, $\mathrm{pH}$ 9.0, containing $0.001 \mathrm{M}$ EDTA and with Antigen Retrieval AR-10 Solution pH 10 (Biogenex, San Ramon, CA, USA), and the same autoclave pretreatment with the two different buffers followed by proteinase $\mathrm{K}$ treatment at room temperature for $10 \mathrm{~min}$ (ChemMate Proteinase $\mathrm{K}$, DakoCytomation). In subsequent experiments, antigen retrieval for all the antibodies tested and the basal cell antibody cocktail (see Results) was carried out in autoclave at $\mathrm{pH} 9.0$ as described earlier.

For the CK5/CK14/p63 antibody cocktail (clones XM26/LL002/4A4 + Y4A3) dilutions of 1:400, 1:400 and 1:1500 were used, respectively. Luminal CK8/18 were detected with a monoclonal antibody (5D3, 1:400, Novocastra). In control experiments, CK8, CK18, CK19 and CK17 were immunostained separately, using monospecific monoclonal antibodies (TS1, 1:800, NeoMarkers; DC10, 1:300, DakoCytomation; RCK108, 1:200, Euro-Diagnostica (Arnhem, Netherlands) and E3, 1:50, Neomarkers, respectively). ER and PR were immunostained via monoclonal antibodies 6F11 (1:400, Novocastra) and PgR 636 (1:1000, DakoCytomation), respectively. Smooth muscle actin was immunostained via monoclonal antibody 1A4 (DakoCytomation). For ER, PR, smooth muscle actin, CK8/18 and CK17, antigen retrieval was carried out as described for the CK5/ CK14/p63 antibody cocktail. For monospecific CK8, CK18 and CK19 antibodies, proteinase K enzymatic pretreatment was carried out after autoclave treatment.

Immunostaining was carried out with a Techmate $500+$ autostainer (DakoCytomation). Endogenous peroxidase was blocked with hydrogen peroxide (ChemMate Peroxidase-Blocking Solution, DakoCytomation) for $3 \times 2 \mathrm{~min} 30 \mathrm{~s}$ followed by $30 \mathrm{~min}$ primary antibody incubation. A Powervision + polymer kit (PowerVision $+{ }^{\mathrm{TM}}$, Immunovision Technologies Co., Brisbane, CA, USA) was used for detection. The reaction was visualized with $\mathrm{DAB}$ chromogen $(2 \times 5 \mathrm{~min}$, Liquid DAB + , DakoCytomation) and enhanced with $0.5 \% \mathrm{CuSO}_{4}$ for $5 \mathrm{~min}$. Hematoxylin (Chemmate hematoxylin, DakoCytomation) was used as a counterstain.

Basal cell carcinoma, skin and normal breast tissue were used as positive controls. For negative 
controls, we omitted the primary antibodies. The specimens were interpreted as positive for CK5/14 if more than $20 \%$ of the neoplastic cells showed cytoplasmic staining and positive for p63 when the staining was nuclear. Two observers (ML and JI) agreed with the interpretation in more than $95 \%$ of the cases. Amplification of the HER-2 oncogene was determined by chromogenic in situ hybridization as described previously. ${ }^{28}$

\section{Results}

Optimization of Immunohistochemical Staining for Basal Phenotype Breast Cancer

Tissue microarrays (consisting of 97 analyzable primary breast tumors) were first used to optimize detection of basal phenotype breast cancer. The two basal cell CKs (CK5 and CK14) did not identify exactly the same tumors when used alone. Among the 97 tumors, there were seven positive for CK5 and six positive for CK14. Two of the CK5-positive tumors were negative for CK14, and one tumor showed the opposite condition. The transcription factor p63 was expressed in the basal cells of normal breast ducts, and was found in three CK5/14negative tumors and in one CK5/14-positive tumor. To ensure detection of basal phenotype breast cancers with high sensitivity, a cocktail of the three antibodies was used in subsequent experiments. The CK5/CK14/p63 antibody cocktail was further validated by comparing the results of antibody cocktail with those from tests with single antibodies. Only one tumor of the 97 showed a discordant result. An example of a CK5- and p63-positive case immunostained via CK5/CK14/p63 antibody cocktail and single antibodies is shown in Figure 1. More example micrographs can be seen in our website http://www.webmicroscope.net/supplements/ LaaksoM.asp.
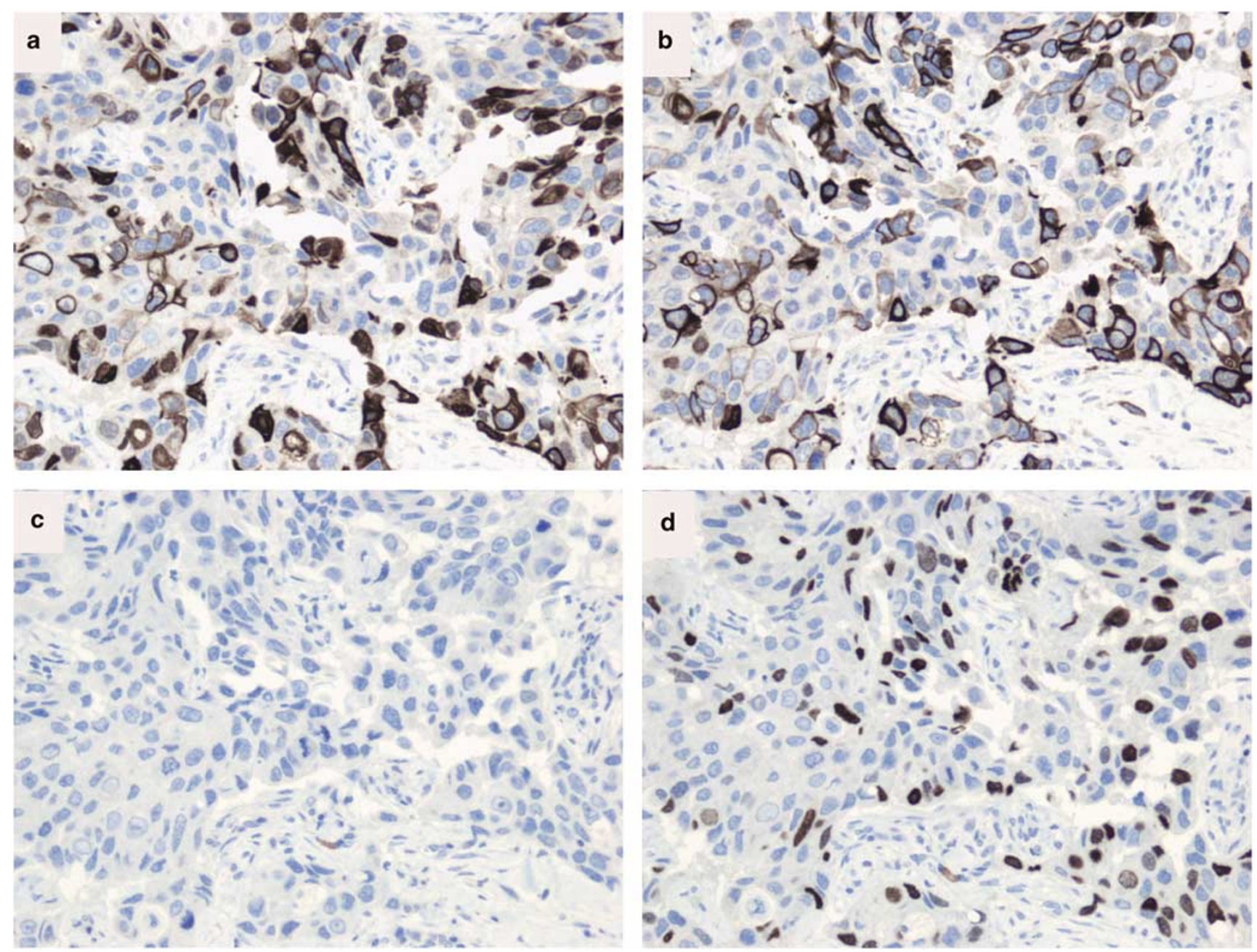

Figure 1 Immunohistochemical staining with CK5/CK14/p63 antibody cocktail (a) shows both cytoplasmic CK and nuclear p63 immunoreactivity. The single antibodies CK5 (b), CK14 (c) and p63 (d) show concordant results with antibody cocktail for this CK5/p63positive tumor. More examples of the immunohistochemistry using the CK5/CK14/p63 antibody cocktail vs single antibodies can be seen as an appendix in our website http://www.webmicroscope.net/supplements/LaaksoM.asp. 


\section{Prevalence and Characterization of Basal Phenotype Breast Cancer}

Of the 288 sporadic invasive ductal breast cancers, $24(9 \%)$ were positive for CK5/14 and $12(4 \%)$ were positive for p63 (Table 1). Matched pairs of primary tumors and metachronous metastases (from 38 patients) were always concordant for CK5/14 expression. There were four CK5/14-positive tumor-metastasis pairs $(11 \%)$ and the rest were negative (examples are shown in the web appendix; http://www.webmicroscope.net/supplements/ LaaksoM.asp).

Of the 288 breast cancers, 207 were immunostained for CK8/18 to investigate the coexpression of basal and luminal CKs. Surprisingly, all tumors in this cohort stained strongly for CK8/18, including all those scored positive for CK5/14 (Table 1). To study luminal CK expression pattern further, we immunostained CK5/14-positive tumors for CK8, CK18 and CK19 separately, using monospecific antibodies. All 20 CK5/14-positive tumors investigated were immunopositive for both CK8 and CK18, although some tumors exhibited relatively weaker staining intensity (Table 2). Staining for CK19 was also positive in all but one of the CK5/14-positive tumors (Table 2). Further characterization of the CK5/14-positive tumors with the myoepithelial differentiation marker smooth muscle actin showed no reactivity in any of the tumors studied.

To ascertain that CK17 does not identify more basal tumors than found with the antibody cocktail CK5/CK14/p63, we stained 110 tumors out of the 288 invasive ductal breast cancers with CK17. These tumors included all the CK5/14-positive tumors and the tissue microarrays used for antibody cocktail staining optimization. There were $11(10 \%)$ CK17positive tumors out of the 110 and only one of the CK17-positive tumors was CK5/14 negative (data not shown). Half of the CK5/14-positive tumors (10/20) showed CK17 positivity (data not shown).

Of the 27 hereditary BRCA1 germline mutation tumors, 21 (78\%) were positive for CK5/14 and one was also positive for p63 (Table 1, see http:// www.webmicroscope.net/supplements/LaaksoM.asp for figures). Out of 20 CK5/14-positive BRCA1associated tumors, 10 were CK8/18 positive with strong intensity $(3+)$, five with moderate intensity $(1-2+)$ and five were totally negative for CK8/18 (data not shown). In contrast to the BRCA1-asso- ciated tumors, only one of the $15 B R C A 2$-associated tumors showed CK5/14 and CK8/18 coexpression, whereas the rest showed the luminal CK5/14negative CK8/18-positive phenotype (Table 1).

\section{Clinicopathological Correlations of the CK5/14-Positive Tumors}

The presence of CK5/14 immunoreactivity showed no correlation with patient age $(P=0.81)$, tumor size $(P=0.42)$ or the presence of axillary lymph node metastasis $(P=0.76$, Table 3$)$. The great majority $(72 \%)$ of the CK5/14-positive tumors were of histological grade $3(P=0.0007)$ and, vice versa, $17 \%$ of the grade III tumors were CK5/14-positive. A strong association was found with negative ER and PR status $(P<0.0001$ for both). As many as $92 \%$ of the CK5/14-positive tumors were ER and PR negative. Positivity for CK5/14 was seen in $12 \%$ of the HER-2 oncogene-amplified cases (amplification confirmed by means of CISH) and in $8 \%$ of the HER-2nonamplified samples $(P=0.59$, Table 3$)$. A total of $24 \%$ of the CK5/14-positive tumors showed HER-2 amplification. The association of CK5/14 and HER-2 was further studied in the subgroup of ER-negative tumors (Table 4). In this group, there was an inverse association, which was statistically highly significant $(P=0.007)$. To confirm further the prevalence of CK5/14 expression and HER-2 amplification, a separate set of 141 sporadic HER-2-amplified breast tumors was studied. There were 19 (13\%) CK5/14positive tumors in this cohort (Table 1). Of these tumors, 116 were stained for CK8/18, including all CK5/14-positive tumors. Confirming the result from

Table 2 Immunoreactivity of luminal CK8, CK18 and CK19 in CK5/14-positive invasive ductal breast cancer

\begin{tabular}{lcrrc}
\hline Luminal CK & Negative & \multicolumn{3}{c}{$\begin{array}{c}\text { Positive } \\
\text { Intensity of the IHC staining }\end{array}$} \\
\cline { 3 - 5 } & & $1+$ & $2+$ & $3+$ \\
\hline CK8 & 0 & $10 \%$ & $10 \%$ & $80 \%$ \\
CK18 & $0 \%$ & $15 \%$ & $0 \%$ & $85 \%$ \\
CK19 & $0 \%$ & $0 \%$ & $5 \%$ & $90 \%$ \\
\hline
\end{tabular}

Data from 20 tumors.

Table 1 Proportion of tumors immunohistochemically positive for basal CK5/14, luminal CK8/18 and the transcription factor p63 in sporadic, sporadic HER-2-amplified and hereditary breast cancers

\begin{tabular}{lccc}
\hline Tumor entity & Positive for CK5/14 & Positive for CK8/18 & Positive for p63 \\
\hline Sporadic ductal tumors (population-based cohort) & $9 \%(25 / 288)$ & $100 \%(207 / 207)$ & $4 \%(12 / 288)$ \\
Sporadic HER-2-amplified tumors (selected cohort) & $13 \%(19 / 141)$ & $100 \%(116 / 116)$ & $8 \%(11 / 141)$ \\
BRCA1-associated tumors & $78 \%(21 / 27)$ & $81 \%(21 / 26)$ & $4 \%(1 / 27)$ \\
BRCA2-associated tumors & $7 \%(1 / 15)$ & $100 \%(15 / 15)$ & $0 \%(0 / 15)$ \\
\hline
\end{tabular}


Table 3 Association of CK5/14 and p63 positivity with clinicopathological features in 288 sporadic invasive ductal breast cancers

\begin{tabular}{|c|c|c|c|c|}
\hline Clinicopathological parameter & CK5/14-positive/total & P-value & p63 positive/total & $\mathrm{P}$-value \\
\hline All invasive ductal tumors & $25 / 288(9 \%)$ & & $12 / 288(4 \%)$ & \\
\hline \multicolumn{5}{|l|}{ Patient age (years) } \\
\hline$<50$ & $5 / 47(11 \%)$ & \multirow{2}{*}{$P=0.81$} & $3 / 47(6 \%)$ & \multirow{2}{*}{$P=0.67$} \\
\hline$\geq 50$ & $20 / 241(8 \%)$ & & $9 / 241(4 \%)$ & \\
\hline \multicolumn{5}{|l|}{ Tumor grade } \\
\hline I & $1 / 19(5 \%)$ & \multirow[t]{3}{*}{$P=0.0007$} & 2/19 (11\%) & \multirow{3}{*}{$P=0.16$} \\
\hline II & 6/163 (4\%) & & $4 / 163(2 \%)$ & \\
\hline III & $18 / 106(17 \%)$ & & $6 / 106(6 \%)$ & \\
\hline \multicolumn{5}{|l|}{ Tumor size $(\mathrm{cm})$} \\
\hline$<2$ & $13 / 120(11 \%)$ & \multirow[t]{3}{*}{$P=0.42$} & $6 / 120(5 \%)$ & \multirow{3}{*}{$P=0.49$} \\
\hline $2-5$ & $11 / 140(8 \%)$ & & $4 / 140(3 \%)$ & \\
\hline$\geq 5$ & $1 / 28(4 \%)$ & & $2 / 28(7 \%)$ & \\
\hline \multicolumn{5}{|l|}{ Axillary lymph node metastasis } \\
\hline No & $17 / 182(9 \%)$ & \multirow[t]{2}{*}{$P=0.76$} & $7 / 182(4 \%)$ & \multirow[t]{2}{*}{$P=0.96$} \\
\hline Yes & 8/106 (8\%) & & $5 / 106(5 \%)$ & \\
\hline \multicolumn{5}{|l|}{$E R$} \\
\hline Negative & $23 / 55(42 \%)$ & \multirow[t]{2}{*}{$P<0.0001$} & $3 / 55(5 \%)$ & \multirow[t]{2}{*}{$P=0.88$} \\
\hline Positive & $2 / 233(1 \%)$ & & $9 / 233(4 \%)$ & \\
\hline \multicolumn{5}{|l|}{$P R$} \\
\hline Negative & $23 / 103(22 \%)$ & \multirow[t]{2}{*}{$P<0.0001$} & $5 / 103(5 \%)$ & \multirow[t]{2}{*}{$P=0.90$} \\
\hline Positive & $2 / 185(1 \%)$ & & $7 / 185(4 \%)$ & \\
\hline \multicolumn{5}{|l|}{ HER-2 amplification } \\
\hline No & $19 / 236(8 \%)$ & $P=0.59$ & $10 / 236(4 \%)$ & \multirow[t]{2}{*}{$P=0.80$} \\
\hline Yes & $6 / 52(12 \%)$ & & $2 / 52(4 \%)$ & \\
\hline
\end{tabular}

Table 4 Association between CK5/14 positivity, HER-2 amplification and ER status in a population-based cohort of 288 invasive ductal breast cancer

\begin{tabular}{lcc}
\hline $\begin{array}{l}\text { CK5/14 } \\
\text { immunoreactivity vs } \\
\text { ER and HER-2 status }\end{array}$ & $\begin{array}{c}\text { CK5/14 negative, } \\
\mathrm{n}=263\end{array}$ & $\begin{array}{c}\text { CK5/14 positive } \\
(\%), \mathrm{n}=25\end{array}$ \\
\hline ER-HER2- & & \\
ER-HER2+ & 12 & $18(60 \%)$ \\
ER+HER2- & 20 & $5(20 \%)$ \\
ER+HER2+ & 205 & $1(0.5 \%)$ \\
& 26 & $1(4 \%)$ \\
\hline
\end{tabular}

$P=0.59$ (HER-2 vs CK5/14 in ER+ and ER- combined).

$P=0.007$ (HER-2 vs CK5/14 in ER- subgroup).

the population-based cohort, all tumors showed strong CK8/18 immunoreactivity (Table 1).

\section{Clinicopathological Correlations Regarding Transcription Factor p63}

In all, $4 \%$ of the invasive ductal breast cancers were p63 positive, but there was no correlation with patient age, tumor grade, tumor size, steroid hormone receptor status, axillary lymph node metastasis or HER-2 oncogene amplification (Table 3). In the cohort of 141 HER-2-amplified tumors, $8 \%$ of the tumors showed p63 expression (Table 1), again with no correlation to ER status.

\section{Discussion}

Antibody cocktails have become increasingly popular in immunohistochemical staining of diagnostic tumor markers. When using a single chromogen, they are technically as easy to use as single antibodies, but can offer significantly more diagnostic information, as has been shown with the P504S/p63 antibody cocktail in prostate cancer. ${ }^{29}$ In breast cancer, CK14 is a major partner of CK5 and both of these are associated with the basal phenotype. ${ }^{1-6,8}$ CK6, which is often used in combination with CK5, using the bi-specific antibody D5/16B $4,{ }^{2}$ is not expressed in normal breast tissue, ${ }^{2,30}$ speaking against its importance as a predominant marker of basal and progenitor cells of the mammary duct. ${ }^{2}$ CK17, also a known partner of CK5, is associated with basal phenotype breast cancer, but it has not been shown to relate to breast progenitor cells. ${ }^{1-5,14}$ Further, only one CK17-positive CK5/14-negative tumor was found among 110 invasive ductal breast cancers in this study. Our results showed that CK5 and CK14 are coexpressed in most tumors, but that tumors expressing CK5 only or CK14 only also exist. 
Somewhat surprisingly, nuclear transcription factor p63, which is another basal cell marker and has also been associated with breast cancer, ${ }^{24-26}$ was expressed only in a minority of CK5/14-positive tumors. However, there were rare p63-positive cases alone without CK5 or CK14. For these reasons, we used a CK5/CK14/p63 antibody cocktail, which recognizes basal phenotype breast cancers specifically, but as widely as possible.

We found that $9 \%$ of invasive ductal breast cancers were CK5/14-positive and that they all coexpressed CK8/18. Clarke et $a l^{31}$ suggested that breast progenitor cells positive for CK5 only, originally described by Boecker et $a l^{2}{ }^{2}$ express low amounts of CK8/18, which is detectable only in frozen sections. When using a highly sensitive peroxidase-polymer-based immunostaining method and optimized antigen retrieval, we found that all sporadic invasive ductal tumors, including those positive for CK5/14, immunostained positively for CK8/18. The high sensitivity of CK8/18 immunodetection was also evident among BRCA1-associated tumors, previously considered as CK5/14 positive and mainly CK8/18 negative, ${ }^{19}$ but which mostly immunostained positively for CK8/18. However, a relatively low staining intensity was found in many cases, which suggest that these tumors might have been scored CK8/18 negative with less sensitive immunohistochemical method. The CK8/18 antibody used (clone 5D3) recognizes CK8 and CK18, and also to some extent CK19. ${ }^{32}$ To further characterize luminal CKs in CK5/14-positive tumors, immunostaining using monospecific CK8, CK18 and CK19 antibodies was carried out. All three luminal CKs were expressed in CK5/14-positive tumors, further confirming the coexpression of basal and luminal CKs in this tumor type. Further, all CK5/14-positive tumors in our study lacked expression of smooth muscle actin, indicating that these tumors are not differentiated towards myoepithelial lineage. Our observations indicate that CK5/14positive invasive ductal tumors originate from glandularly committed progenitor cells of the breast, which have been shown to coexpress CK5/14 and CK8/18 during maturation to fully differentiated luminal cells. ${ }^{2,8}$

Cancers associated with the BRCA1 germline mutation have been shown to be associated strongly with basal phenotype breast cancer. ${ }^{18-20}$ This was also the finding in our material, as $78 \%$ of the BRCA1-associated tumors expressed CK5/14. In contrast to sporadic cancer, there were some CK5/ 14-positive CK8/18-negative phenotypes among the BRCA1-associated tumors, suggesting that these tumors probably originate from true basal phenotype cells. Foulkes et al has hypothesized that the key function of the wild-type BRCA1 is to act as a stem cell regulator and promote the differentiation towards glandular epithelium in the normal breast. In BRCA1 mutated tumors, this transition has failed or not completed and basal cell phenotype gene expression is retained. ${ }^{33}$ This kind of incidents might lead to CK5/14 positive but CK8/18 negative or weakly these luminal CKs expressing BRCA1 germline-mutated tumors seen in this study. However, BRCA1-associated tumors were found to be a more heterogeneous group than previously thought. Although CK5/14 positivity was a common finding, there were several tumors resembling sporadic 'luminal-type' tumors in their CK profile (CK5/ 14-; CK8/18 + ). The results from BRCA2 germline mutation carriers were more uniform and showed that their breast tumors were of luminal epithelium phenotype, similar to the majority of sporadic cancers.

Our results showed that CK5/14-positive breast cancers were exclusively ER- and PR-negative grade III carcinomas, which is in line with the results of earlier studies, ${ }^{4,7,9,10,16-18}$ This finding could suggest a link to HER-2 oncogene amplification, which has also been associated with negative ER status in a large number of studies (Konecny et $a l^{34}$ and references therein). Unexpectedly, our results showed that there was no statistically significant association between CK5/14 positivity and HER-2 amplification (CISH) when both ER-positive and ERnegative tumors were considered. However, there was a significant inverse association between HER-2 oncogene amplification and CK5/14 immunopositivity within the subgroup of ER-negative tumors. This indicates that CK5/14-positive breast cancers and tumors with HER-2 amplification are mainly different tumor entities. Published data on HER-2 and basal phenotype are limited and somewhat conflicting. The results of microarray studies have suggested that basal phenotype breast cancer is HER-2 nonamplified. ${ }^{10,17,18}$ In contrast, Birmbaum et $a l^{9}$ have reported that basal phenotype breast cancer is associated with HER-2 amplification. Our results clearly show that HER-2-amplified CK5/14-positive breast cancers exist, although it is a minority. A recent in vitro study suggests that these tumors may form an interesting subgroup of HER-2-positive tumors, for example, with respect to therapeutic sensitivity to the HER-2-inhibiting drug trastuzumab. ${ }^{35}$ Both CK5/14 positivity and HER-2 amplification are each associated with poor clinical outcome, ${ }^{17,18,26}$ but currently there are no data available on prognosis or therapeutic response prediction among patients whose tumors are HER2 amplified and positive for basal CKs.

In conclusion, CK5/14-positive breast cancers represent about $9 \%$ of sporadic invasive ductal breast cancers and $78 \%$ of BRCA1-associated tumors. These tumors are mostly aggressive grade III steroid hormone receptor-negative breast cancers, and they are inversely associated with HER-2 oncogene amplification in the subgroup of ERnegative tumors. These tumors express basal and luminal CKs concomitantly and therefore may originate from luminally committed progenitor cells of the breast. 


\section{Acknowledgements}

We thank Ms Ritva Kujala, Ms Helvi Salmela, Ms Pirjo Pekkala, Ms Päivi Kärki, Ms Sari Toivola and Ms Eeva Riikonen for skillful technical assistance. This study was financially supported by grants from the Pirkanmaa Hospital District Research Foundation, Medical Research Fund of Seinäjoki Central Hospital, Sigrid Juselius Foundation, the Finnish Cancer Foundation and Algol-Award.

\section{References}

1 Moll R, Franke WW, Schiller DL. The catalog of human cytokeratins: patterns of expression in normal epithelia, tumors and cultured cells. Cell 1982;31:11-24.

2 Boecker W, Moll R, Poremba C, et al. Common adult stem cells in the human breast give rise to glandular and myoepithelial cell lineages: a new cell biological concept. Lab Invest 2002;82:737-745.

3 Chu PG, Weiss LM. Keratin expression in human tissues and neoplasms. Histopathology 2002;40: 403-439.

4 Malzahn K, Mitze M, Thoenes M, et al. Biological and prognostic significance of stratified epithelial cytokeratins in infiltrating ductal breast carcinomas. Virchows Arch 1998;433:119-129.

5 Wetzels RHW, Kuijpers HJH, Lane EB, et al. Basal cellspecific and hyperproliferation-related keratins in human breast cancer. Am J Pathol 1991;138:751-763.

6 Abd El-Rehim DM, Pinder SE, paish CE, et al. Expression of luminal and basal cytokeratins in human breast cancer. J Pathol 2004;203:661-671.

7 Korsching E, Packeisen J, Agelopoulos K, et al. Cytogenetic alterations and cytokeratin expression patterns in breast cancer: integrating a new model of breast differentiation into cytogenetic pathways of breast carcinogenesis. Lab Invest 2002;82: 1525-1533.

8 Boecker W, Buerger H. Evidence of progenitor cells of glandular and myoepithelial cell lineages in the human adult female breast epithelium: a new progenitor (adult stem) cell concept. Cell Prolif 2003;36:73-84.

9 Birmbaum D, Bertucci F, Ginestier C, et al. Basal and luminal breast cancer: basic or luminous? Int J Oncol 2004;25:249-258.

10 Perou CM, Sorlie T, Eisen MB, et al. Molecular portraits of human breast tumours. Nature 2000; 406:747-752.

11 Bánkfalvi A, Ludwig A, de-Hesselle B, et al. Different proliferative activity of the glandular and myoepithelial lineages in benign proliferative and early malignant breast diseases. Mod Pathol 2004;17:1051-1061.

12 Boecker W, Moll R, Dervan P, et al. Usual ductal hyperplasia of the breast is a committed stem (progenitor) cell lesion distinct from atypical ductal hyperplasia and ductal carsinoma in situ. J Pathol 2002;198:458-467.

13 Otterbach F, Bànkfalvi À, Berhner S, et al. Cytokeratin $5 / 6$ immunohistochemistry assists the differential diagnosis of atypical proliferations of the breast. Histopathology 2000;37:232-240.

14 van de Rijn M, Perou CM, Tibshirani R, et al. Expression of cytokeratins 17 and 5 identifies a group of breast carcinomas with poor clinical outcome. Am J Pathol 2002;161:1991-1996.

15 Gordon LA, Mulligan KT, Maxwell-Jones $\mathrm{H}$, et al. Breast cell invasive potential relates to the myoepithelial phenotype. Int J Cancer 2003;106:8-16.

16 Nielsen TO, Hsu FD, Jensen $\mathrm{K}$, et al. Immunohistochemical and clinical characterization of the basal-like subtype of invasive breast carcinoma. Clin Cancer Res 2004;10:5367-5374.

17 Sorlie T, Perou CM, Tibshirani R. Gene expression patterns of breast carcinomas distinguish tumor subclasses with clinical implications. Proc Natl Acad Sci USA 2001;98:10869-10874.

18 Sorlie T, Tibshirani R, Parker J, et al. Repeated observation of breast tumor subtypes in independent gene expression data sets. Proc Natl Acad Sci USA 2003;100:8418-8423.

19 Foulkes WD, Brunet J-S, Stefansson IM, et al. The prognostic implication of the basal-like (Cyclin $\mathrm{E}^{\text {high/ }}$

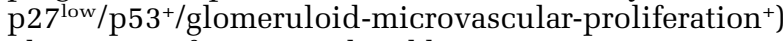
phenotype of BRCA1-related breast cancer. Cancer Res 2004;64:830-835.

20 Foulkes WD, Stefansson IM, Chappuis PO, et al. Germline BRCA1 mutations and a basal epithelial phenotype in breast cancer. J Natl Cancer Inst 2003;95:1482-1485.

21 Barbareschi M, Pecciarini L, Cangi MG, et al. p63, a p53 homologue, is a selective nuclear marker of myoepithelial cells of the human breast. Am J Surg Pathol 2001;25:1054-1060.

22 Nylander K, Vojtesek B, Nenutil R, et al. Differential expression of p63 isoforms in normal tissues and neoplastic cells. J Pathol 2002;198:417-427.

23 Wang X, Mori I, Tang W, et al. p63 expression in normal, hyperplastic and malignant breast tissues. Breast Cancer 2002;9:216-219.

24 Ribeiro-Silva A, Zambelli Ramalho LN, Britto Garcia S, et al. The relationship between p63 and p53 expression in normal and neoplastic breast tissue. Arch Pathol Lab Med 2003;127:336-340.

25 Reis-Filho JS, Simpson PT, Martins A, et al. Distribution of p63, cytokeratin 5/6 and cytokeratin 14 in 51 normal and 400 neoplastic human tissue samples using TARP-4 multi-tumor tissue microarray. Virchows Arch 2003;443:122-132.

26 Makretsov NA, Huntsman DG, Nielsen TO, et al. Hierarchical clustering analysis of tissue microarray immunostaining data identifies prognostically significant groups of breast carcinoma. Clin Cancer Res 2004;10:6143-6151.

27 Loman N, Johannsson O, Kristoffersson U, et al. Family history of breast and ovarian cancers and BRCA1 and BRCA2 mutations in a population-based series of early-onset breast cancer. J Natl Cancer Inst 2001;93:1215-1223.

28 Isola J, Tanner $\mathrm{M}$, Forsyth $\mathrm{A}$, et al. Interlaboratory comparison of HER-2 oncogene amplification as detected by chromogenic and fluorescence in situ hybridization. Clin Cancer Res 2004;10: 4793-4798.

29 Tacha DE, Miller RT. Use of p63/P504S monoclonal antibody cocktail in immunohistochemical staining of prostate tissue. Appl Immunohistochem Mol Morphol 2004;12:75-78.

30 Moll R. Cytokeratins as markers of differentiation in the diagnosis of epithelial tumors. Subcell Biochem 1998;31:205-262. 
31 Clarke CL, Sandle J, Parry SC, et al. Cytokeratin 5/6 in normal human breast: lack of evidence for a stem cell phenotype. J Pathol 2004;204:147-152.

32 Angus B, Purvis J, Stock D, et al. NCL-5D3: a new monoclonal antibody recognizing low molecular weight cytokeratins effective for immunohistochemistry using fixed paraffin-embedded tissue. J Pathol 1987;153:377-384.

33 Foulkes WD. BRCA1 functions as a breast stem cell regulator. J Med Genet 2003;41:1-5.
34 Konecny G, Pauletti G, Pegram M, et al. Quantitative association between HER-2/neu and steroid hormone receptor in hormone receptor positive primary breast cancer. J Natl Cancer Inst 2003;95: 142-153.

35 Tanner M, Kapanen AI, Junttila T, et al. Characterization of a novel cell line established from a patient with Herceptin-resistant breast cancer. Mol Cancer Ther 2004;3:1585-1592. 